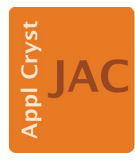

JOURNAL OF APPLIED CRYSTALLOGRAPHY

ISSN 1600-5767

Received 16 July 2015

Accepted 29 October 2015

Edited by S. Sasaki, Tokyo Institute of Technology, Yokohama, Japan

Keywords: Debye function analysis; powder diffraction data processing; nanocrystalline materials; disorder; computer programs.

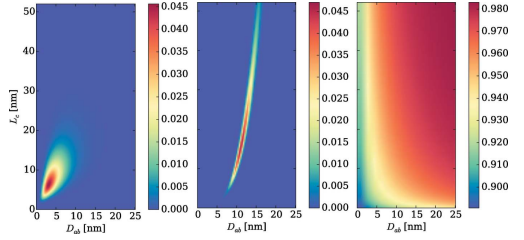

C 2015 International Union of Crystallography

\section{DEBUSSY 2.0: the new release of a Debye user system for nanocrystalline and/or disordered materials}

\author{
Antonio Cervellino, ${ }^{a}$ Ruggero Frison, ${ }^{\mathrm{b}, \mathrm{c} *}$ Federica Bertolotti ${ }^{\mathrm{d}}$ and Antonietta \\ Guagliardi $^{\mathrm{c}}$
}

\begin{abstract}
${ }^{a}$ Swiss Light Source, Paul Scherrer Institut, 5232, Villigen, Switzerland, bUniversity of Zurich, Winterthurerstrasse 190, $\mathrm{CH}-8057$ Zurich, Switzerland, 'Istituto di Cristallografia and To.Sca.Lab, National Council of Research, via Valleggio 11, Como, 22100, Italy, and ' Dipartimento di Scienza e Alta Tecnologia and To.Sca.Lab, Università dell'Insubria, via Valleggio 11, Como, 22100, Italy. *Correspondence e-mail: ruggero.frison@uzh.ch
\end{abstract}

The new release of DEBUSSY is introduced, a free open-source package devoted to the application of the Debye function analysis of powder diffraction data from nanocrystalline, defective and/or nonperiodic materials. The general strategy of the suite remains unchanged, following a two-step approach managed by the CLAUDE and DEBUSSY programs, respectively. The first step essentially consists in generating a database where structural, size and shape information on a nanocrystal population is stored; the second step consists in the calculation, through the Debye scattering equation, of the total diffraction pattern using the previously generated database and a set of model parameters provided by the user and then optimized by the program. The novelties lie in the computational, modelling and graphical levels, and several new programs and features have been added. Among these are a new general comprehensive input file format (.ddb) for the database generation, the automatic management of the space-group symmetry and .cif file, new nanocrystal shapes, size-dependent site occupancy factors and thermal parameters for each atomic species, new lattice expansion functions, and a newly developed algorithm for calculating the standard errors of the optimized parameters. The CLAUDE suite also includes a program for calculation of the pair distribution function. Last but not least, a graphical user interface, which makes it easier to edit input files, execute the programs of the suite in a chain-like way, and plot the results in an automatic or custom manner, is provided.

\section{Introduction}

The ever growing technological interest in nanostructured materials is increasingly emphasizing the importance of powerful and reliable analytical techniques for their characterization on both the experimental and the computational side.

Total scattering techniques, like the pair distribution function (PDF) (Egami \& Billinge, 2003) analysis and Debye function analysis (DFA) (Cervellino et al., 2003), are establishing themselves to this aim as an (often better) alternative to conventional diffraction techniques based on Rietveld-like methods (Rietveld, 1969; Cheary \& Coelho, 1992). From this perspective, the specific goal of the structural analysis of a nanoscaled material through diffraction techniques is to yield quantitative information on the atomic structure, in terms of its most relevant parameters like stoichiometry and phase composition, structural defects, strain and lattice distortion, surface features, and, additionally, size, shape and their distributions. 
Table 1

Comparison between CLAUDE 1.0 and CLAUDE 2.0.

The output file type of some programs is reported in square brackets. For details refer to $\$ 3.1$.

\begin{tabular}{lll}
\hline Task & CLAUDE 1.0 & CLAUDE 2.0 \\
\hline General input & DB_PHASE_Info & .ddb file \\
& DB_CLU_Info & \\
Phase information & .pha file & .Cif or .pha file \\
Internal input file preparation & - & DB_PHA_CLU program \\
Space-group calculation & MK_GROUP program & Discontinued \\
Unit-cell content calculation & MK_CELL program & Two improvements \\
Spherical (SPH) NC & MK_SPHERE program [.xyz] & MK_BALL program [.xyz, .smp] \\
Cubic (QBE) NC calculation & - & MK_QBE program [.xyz, .smp] \\
Parallepipedic (PAR, HEX, CYL) NC & MK_RODS program [.xyz] & MK_RODS program [.xyz] \\
Parallepipedic (PAR, HEX, CYL) NC calculation & MK_LAYERS program [.smp] & MK_LAYER_GEN program [.smp] \\
Monoclinic phase NC calculation & - & MK_LAYER_OBL program [.smp] \\
User-defined cluster shape, l.p. and building block & - & MK_LATSET program [.xyz, .smp] \\
Interatomic distance calculation & MK_DIST program [.dist] & Discontinued \\
Interatomic distance sampling & MK_SAMP program [.smp] & Discontinued \\
Interatomic distance calculation and sampling & MK_DISSSAM program & Discontinued \\
Noncrystalline phase cluster calculation & MK_MOLEC program [.smp] & Output harmonized \\
Single NC or cluster pattern calculation & MK_PATTERN program & Extended \\
$G(r)$ calculation & - & MK_G_OF_R program \\
Graphical user interface & - & Yes \\
\hline
\end{tabular}

mics (Guagliardi et al., 2010; Delgado-López et al., 2014) and metal-organic compounds (Cervellino et al., 2012; Bertolotti et al., 2014)]. The general strategy of the suite has remained unchanged. The novelties lie in the computational, modelling and graphics levels. The manuscript is organized as follows: the main features of the DEBUSSY suite are initially recalled in $\S 2$, along with the main differences between the former and new releases. Then details about the upgrades on the computational and modelling sides of the new release are described in $\$ 3$, including details about the graphical user interface (GUI) in $\$ 3.3$.
The Debye scattering equation (DSE) (Debye, 1915; Cervellino et al., 2010) provides the differential elastic scattering cross section (or the powder diffraction pattern) of a sample from the distribution of the interatomic distances $d_{i j}$ without any assumption on periodicity and order:

$$
\begin{aligned}
I(q)= & \sum_{s=1}^{N_{\mathrm{a}}} N_{s} f_{s}^{2}(q) o_{s}^{2}+2 \sum_{s=1}^{N_{\mathrm{a}}} f_{s}^{2}(q) T_{s}^{2}(q) o_{s}^{2} \sum_{i>j=1}^{N_{s}} \frac{\sin \left(2 \pi q d_{i j, s s}\right)}{2 \pi q d_{i j, s s}} \\
& +2 \sum_{s>s^{\prime}=1}^{N_{\mathrm{a}}} f_{s}(q) f_{s^{\prime}}(q) T_{s}(q) T_{s^{\prime}}(q) o_{s} o_{s^{\prime}} \sum_{i=1}^{N_{s}} \sum_{j=1}^{N_{s^{\prime}}} \frac{\sin \left(2 \pi q d_{i j, s s^{\prime}}\right)}{2 \pi q d_{i j, s s^{\prime}}} .
\end{aligned}
$$

Here $N_{\mathrm{a}}$ is the number of atomic species and $N_{s}$ the number of atoms of the $s$ th species $\left(s=1 \ldots N_{\mathrm{a}}\right)$. Each atom of the same species is identified by a mean position vector $r_{s i}$ such that $d_{i j, s s}=\left|r_{s i}-r_{s j}\right|$, a mean occupancy factor $o_{s}$, a (radiationdependent) scattering length $f_{s}$ and an isotropic Debye-Waller factor $T_{s}$. Thus the DSE is a powerful tool for quantitatively determining, with a physically and chemically based model, the structural properties of both isotropically and anisotropically shaped nanoparticles with very small coherent domains and/or a high defect concentration. In addition, unlike the PDF, the DSE does not transform the experimental data, thus keeping the advantage of simultaneously dealing with both reciprocal and direct spaces.

In this paper we introduce the second release of DEBUSSY (Cervellino et al., 2010), an open-source software package implementing the Debye equation for the analysis of powder diffraction data from nanocrystalline, defective and/or nonperiodic materials. DEBUSSY has been so far successfully applied in modelling different kinds of nanomaterials [metals (Cervellino et al., 2004), oxides (Cernuto, Masciocchi et al., 2011; Cernuto, Galli et al., 2011; Frison et al., 2013), biocera-

\section{The DEBUSSY suite}

Here we recall the working strategy and the general features of the DEBUSSY suite (Cervellino et al., 2010). The $D E B U S S Y$ approach is of bottom-up type and consists of two main steps respectively managed by CLAUDE (a suite of 12 programs, see Table 1) and DEBUSSY (a single program taking the name of the package). The first step (I) mainly consists in generating a mono- or bivariate population of nanocrystals (NCs) with increasing size and desired shape using the unit cell as calculated from the asymmetric unit and the space-group operators, or as provided by user input (according to the required file format). In order to optimize the execution of step I, some workarounds are used, like for example grouping the same distances in a single term and evaluating its multiplicity, exploiting the crystal symmetry in order to reduce the number of interatomic distances (Grover \& McKenzie, 2001; Cervellino \& Guagliardi, 2010; Thomas, 2010), and sampling the NCs' interatomic distances (Hall \& Monot, 1991; Navaza, 2002; Cervellino et al., 2006). Table 1 summarizes the main differences and correspondences in the former and new releases of CLAUDE.

In the second step (II) of the approach, which is managed by the single program DEBUSSY, the sampled distance sets previously calculated by $C L A U D E$ are evaluated as powder diffraction patterns and compared with experimental data. The optimal similarity is sought by varying some parameters [e.g. mixing coefficients, lattice expansions, Debye-Waller and site occupancy (s.o.f.) factors etc.] that modify the calculated pattern. The diffraction pattern is interpreted by DEBUSSY as a weighted sum of the patterns of the NCs represented in the database, according to their number fraction of a lognormal size distribution. Size distributions constrain the mixing coefficients of all the individual NC sizes in each phase by forcing them to follow an analytical (lognormal) function. 
Table 2

Comparison between DEBUSSY 1.0 and DEBUSSY 2.0.

For details refer to $\$ 3$.

\begin{tabular}{lll}
\hline Input/output & DEBUSSY 1.0 & DEBUSSY 2.0 \\
\hline $\begin{array}{l}\text { General input } \\
\text { Phase parameters }\end{array}$ & $\begin{array}{l}\text {.dwa file } \\
\text {.par file }\end{array}$ & $\begin{array}{l}\text {.dwa file } \\
\text {.par file: modified and } \\
\text { extended }\end{array}$ \\
Refinement strategy & .ref file & $\begin{array}{c}\text { ref file: refinement flags } \\
\text { modified }\end{array}$ \\
Output matrix & .mtx file & .mtx file: extended \\
$\begin{array}{l}\text { S.u. matrix } \\
\text { Lattice expansion }\end{array}$ & - & .mtxe file \\
Occupancy factors & One global & $\begin{array}{l}\text { Five models } \\
\text { Atom specific and size } \\
\text { dependent }\end{array}$ \\
Thermal parameters & One global & $\begin{array}{c}\text { Atom specific and size } \\
\text { dependent }\end{array}$ \\
Background & One experimental & Multiple experimental \\
& and Chebyshev & and Chebyshev \\
& polynomials & polynomials \\
Total pattern simulation & - & Yes \\
Graphical user interface & - &
\end{tabular}

Simplex (Nelder \& Mead, 1965) or simulated annealing (Metropolis et al., 1953) algorithms are used for seeking the best similarity. A single-shot (simulation) mode is also implemented. In contrast to the first version of DEBUSSY, not only the (number- and mass-weighted) average sizes and (possibly bivariate) size dispersions of the NC population are given as the result, but also fully size-dependent profiles of lattice expansion/contraction, atomic site occupation factors and thermal parameters. As in version 1.0 the number of terms in the DSE is reduced by many orders of magnitude without losing accuracy and the Chebyshev recursion-aided fast Debye summation further speeds up the calculation (Cervellino et al., 2006; Cervellino \& Guagliardi, 2010).

DEBUSSY requires a main input (.dwa) file containing information on the experimental data set(s), the database(s) storing the population(s) of NCs/clusters and the names of further files giving the refinement details. Parameters (for each phase) with their lower and upper bounds and refinement flags (for each parameter) are provided by additional external (.par and .ref) files. Table 2 summarizes the main differences and correspondences in the 1.0 and 2.0 releases of DEBUSSY.

\section{DEBUSSY 2.0}

With the 2.0 release of DEBUSSY new features have been introduced in both the CLAUDE and the DEBUSSY parts, as summarized in Tables 1 and 2 .

\subsection{CLAUDE 2.0}

The CLAUDE 2.0 suite includes a total of 13 programs and, as schematically shown in Fig. 1, mainly builds databases of .smp files, storing the information on the multiplicity of sampled interatomic distances between pairs of atoms in NCs or other kinds of nanoclusters. Additionally, for each .smp file an associated .smp_INFO file is generated, containing important information about the detailed cluster composition. This has now been extended and completed with other information on nanocrystal size, lattice parameters, number of growth directions (for NCs) and number of lattice points. Fig. 1 shows a flow chart of the CLAUDE suite.

The database generation can, therefore, be performed by a step-by-step procedure where the user invokes the various programs at a terminal input or using the GUI included in the program package.

3.1.1. New input files and programs. A new comprehensive single input file, .ddb, has been implemented, which allows the user to accomplish step I by simply selecting the file containing the phase information and the options for the choice of the particle shape and maximum size, plus some resolution-related ones like the wavelength and maximum $2 \theta$ angle. Fully automatized space group and symmetry operator management has also been implemented. As previously mentioned for the 1.0 release, depending on whether a crystalline or a noncrystalline phase is being handled, the phase information file has to be provided by two different file formats: in the first case a .pha file containing the necessary information (phase name, unitcell parameters, space-group number, asymmetric unit content); in the second a .xyz file with the list of Cartesian

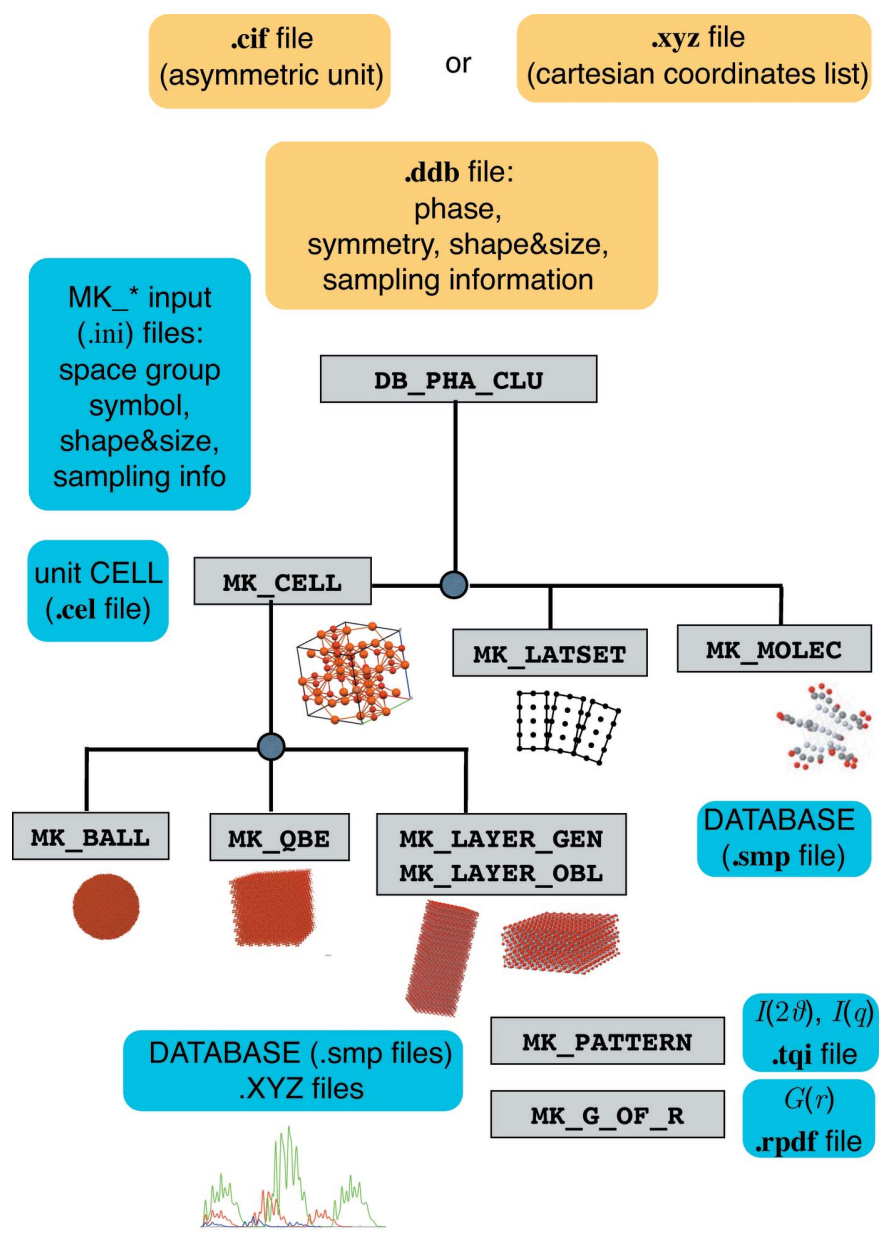

Figure 1

A flow chart of the CLAUDE suite, illustrating the step-by-step database generation procedure. Light orange fields indicate mandatory user input files, blue fields program output files and grey fields single programs of the suite. Circles highlight the main decision tasks. 
atomic coordinates. A new utility, CIF2PHA, has been included in order to convert the phase information reported in the .cif (crystallographic information file) into the internal standard .pha file format. .cif files downloadable from several crystallographic databases (Cambridge Structural Database, Inorganic Crystal Structure Database, American Mineralogist, Crystallography Open Database) are managed.

In order to handle the .ddb general input file the new program DB_PHA_CLU has been included, which prepares all the required inputs for the other programs of the suite so that they can be chain executed according to the user-defined workflow, as specified in the .ddb file. The programs dealing with nanoparticle calculations have been extended, fully revised and optimized, as detailed in the following:

(1) MK BALL. This program builds spherical NCs selecting a set of lattice points (l.p.s) included in spheres. It deals with any lattice whose vectors are read from the .cel file (see Fig. 1). Like its predecessor MK_SPHERE, families of NCs of increasing size are built up to a maximum defined by the user, where the $n$th cluster contains exactly $n^{3}$ l.p.s. A smart strategy of partial occupancy of the 1.p.s near the surface is used to preserve shape, 1.p. count and sample stoichiometry. Multiplicityweighted distances between l.p.s are then convoluted with the Patterson function ${ }^{1}$ of the given unit cell to yield directly all the interatomic distances in the most efficient way. Optional outputs include

(a) the generation of direct space (.xyz) atomic coordinate files for each cluster;

(b) the generation of either all the clusters up to the given maximum size (optionally with a tunable step) or only the largest sphere.

(2) MK_QBE. This program is the 'cubic analog' of MK_BALL described above, meaning that perfect cubes instead of spheres are built. The keywords and output are similar.

(3) MK_LAYER_GEN. This program is similar to its formerly distributed version MK_LAYERS, in that it calculates bivariate families of NCs with different base diameters and different heights perpendicular to the base. However, it implements a fully revised and optimized algorithm and the option of generating the largest $\mathrm{NC}$ introduced for MK BALL and MK_QBE. Note that, in this case, the user choice is limited to the base diameter size, while different lengths are always produced owing to the intrinsic algorithm used.

(4) MK_LAYER_OBL. This program is similar to the MK_LAYER_GEN program described above, but it builds NCs where the height is not necessarily orthogonal to the base, as in the case of monoclinic and triclinic structures. This feature makes the program necessarily twice as slow as MK_LAYER_GEN, although it is more capable - hence the apparent duplication.

(5) MK LATSET. This program works on a similar principle to all the previous NC builders except that it calculates only a single cluster at each run, and the set of 1.p.s. enclosed by a geometrical surface, as well as the file containing information

\footnotetext{
${ }^{1}$ The Patterson function is here intended as the set of distinct interatomic distances between atoms in the unit cell, together with their multiplicity, rather than the set of interatomic vectors.
}

on the 'building block' associated with each 1.p., is now read from external user-generated files. Therefore the 1.p.s are better described as a 'translation point set', as they may not belong to any lattice. Once more the interatomic distances are calculated as a convolution of the weighted lattice distances with the Patterson function of a building block.

(6) MK_G_OF_R. This program calculates the radial distribution function $G(r)$ starting from $2 \theta$ intensity data files or directly a precomputed $S(q)$ for different kinds of radiation (X-rays, electrons and neutrons). Additionally, the radial coordinate minimum, maximum and step, normalization mode, and scaling factors can be chosen. Details are given in the manual.

All the NC builders (MK_BALL, MK_QBE, MK_LAYER_GEN, MK_LAYER_OBL) present new common features, namely (i) a new format (.cely, see the manual for details) describing the content of the unit cell, which is particularly suited for molecular NCs, is available as an alternative to the standard (.cel) one, and (ii) isotropic and two-dimensional paracrystallinity models have been added and can be used to generate defective NCs, following Welberry's approach (Welberry, 2010) and as described by Cervellino et al. (2012). All the new keywords, mandatory and optional, are described in the new CLAUDE manual.

3.1.2. Modified programs. (1) MK_CELL. Two main improvements have been made: (i) the symmetry handling has been fully automatized, such that the space-group operations are now read by files stored in an external database (instead of being generated in advance by the user); (ii) the (optional) construction of the most symmetric unit cell around a suitably chosen origin starting from the user-supplied asymmetric unit is possible.

(2) MK_MOLEC. For this program the output has been harmonized according to the rest of the CLAUDE suite.

(3) MK_PATTERN. The option to produce not only the powder diffraction pattern for a single cluster but also the corresponding $S(q)$ with different scaling modes, as described in the manual, has been added.

3.1.3. Superseded programs. Some of the CLAUDE 1.0 programs have been discontinued. The functionality of MK_GROUP has been now eliminated as redundant (see above). The interatomic distance sampling algorithm, formerly handled through the MK_SAMP and MK_DIST programs, is now dealt with internally by each $\mathrm{NC}$ builder.

\subsection{DEBUSSY 2.0}

For the DEBUSSY part the most relevant new features are the following:

(1) The site occupancy factors and isotropic Debye-Waller factors of each atom in the structural model are now managed and can be refined according to a constant-size or a sizedependent law, by adjusting one or three parameters, respectively (an example is given in Fig. 2). These features have been thoroughly tested and have given very interesting and robust results (see Frison et al., 2013; Delgado-López et al., 2014; Cervellino et al., 2014). 


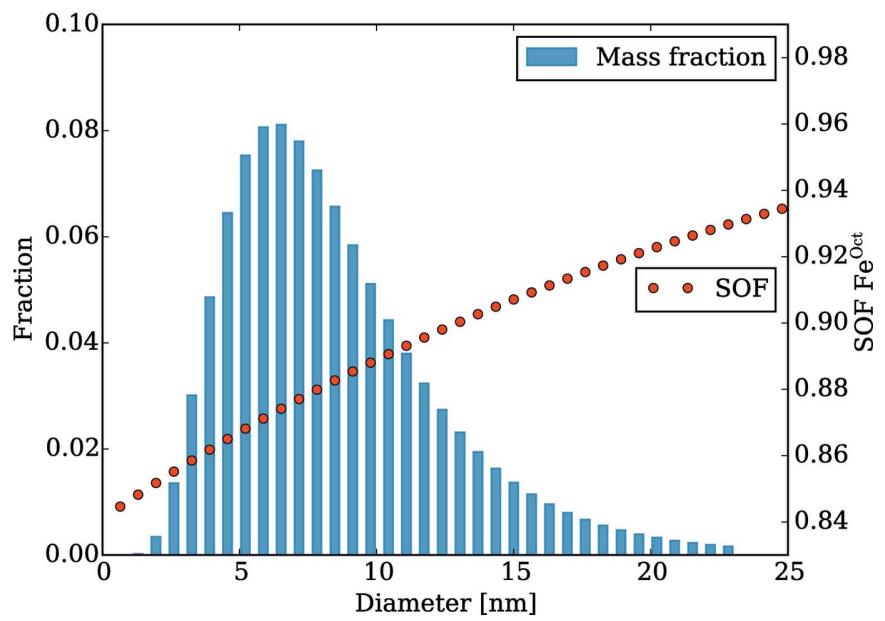

(a)

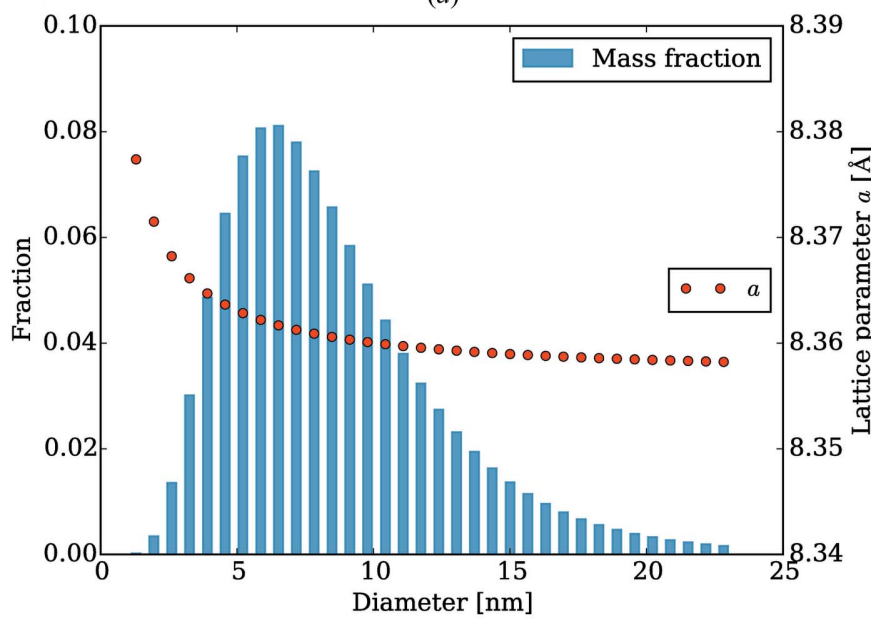

(b)

Figure 2

Nanoparticle mass fraction distribution with (a) s.o.f. of Fe octahedral site and $(b)$ lattice parameter as a function of nanoparticle size for maghemite samples. For details see Frison et al. (2013).

(2) Size(s) and standard deviation(s) in the .par file for both the lognormal and the bivariate lognormal distribution functions describe now the true (number-based) average values ${ }^{2}$ in nanometres.

(3) Three additional size-dependent lattice expansion functions (making a total of five) are available.

(4) The .par file has been reorganized in order to manage parameters related to the atomic species; additionally, refinement flags in this file can be used to control the refinement of each single parameter, while refinement flags in the .ref file can be used to control groups of parameters (size, lattice expansion, s.o.f. and Debye-Waller factor of each atom) and multistage refinement strategies.

(5) Compton scattering is calculated according to the sample composition and added as an additional component to the total scattering model.

\footnotetext{
${ }^{2}$ In the 1.0 release the size-related parameters appearing in the .par file were centre(s) and width(s) of the size distribution.
}

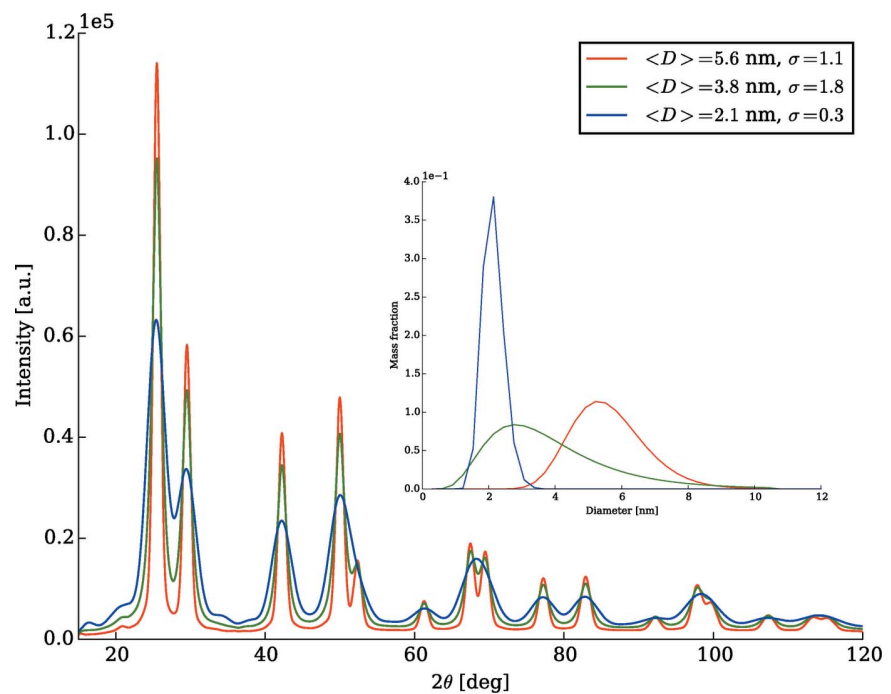

Figure 3

Calculated total scattering patterns and mass distributions (inset) of $\mathrm{Pt}$ spherical nanoparticles with different average diameter and/or standard deviation of the lognormal size distribution.

(6) More than one blank curve can be provided to the program and used as additional model components, all being suitably scaled by linear least squares.

(7) The simulated diffraction pattern from a population of NCs and according to the given input parameters can be calculated without needing an experimental data file, as shown in Fig. 3, displaying three diffractions patterns for three different sets of size parameters.

(8) The calculation of the standard errors of the optimized parameters and of their most relevant functions is implemented using numerical first and second derivatives of the $\chi^{2}$ at the computed minimum to evaluate the variance-covariance matrix, based on a robust pseudo-inversion algorithm that takes into account possible parameter correlations. This, in turn, enables also the propagation of the errors to derived quantities, which are evaluated starting from the refined parameters. The output has now been completed with the standard deviations for the refined parameters and the principal derived quantities.

\subsection{Graphical user interface}

The GUI allows the user to prepare the input files either from scratch or by loading and editing preexisting ones, to generate any of the output files by clicking on the corresponding button, and to display the results as predefined or customized plots. It consists of a single notebook window with two main tabs, one dedicated to CLAUDE 2.0 and the other to $D E B U S S Y$ 2.0, which will in the following be referred to as $C L A U D E_{-} G U I$ and $D E B U S S Y \_G U I$, respectively.

Fig. 4 shows the CLAUDE_GUI window, which is organized into four tabs dedicated to the input file editing for the main CLAUDE 2.0 tasks, and a vertical side field where the buttons corresponding to the main tasks are located. In this way the functionality of $C L A U D E 2.0$ is fully disclosed to the 


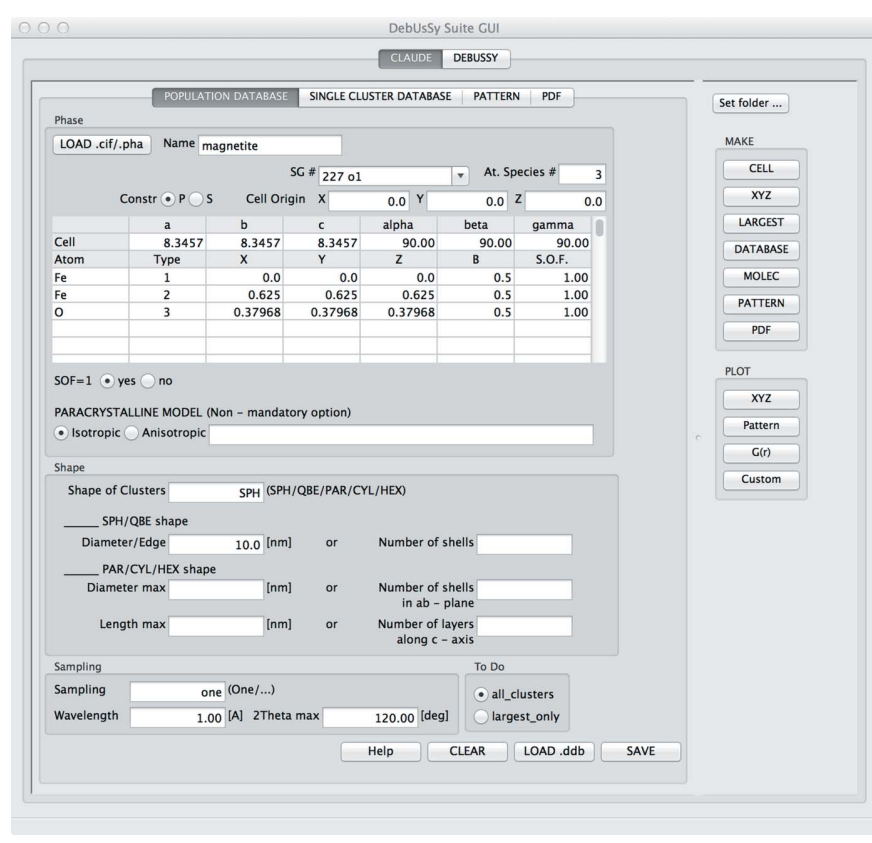

Figure 4

The CLAUDE_GUI window with the editor of the main input file (.ddb) for database generation from an asymmetric unit.

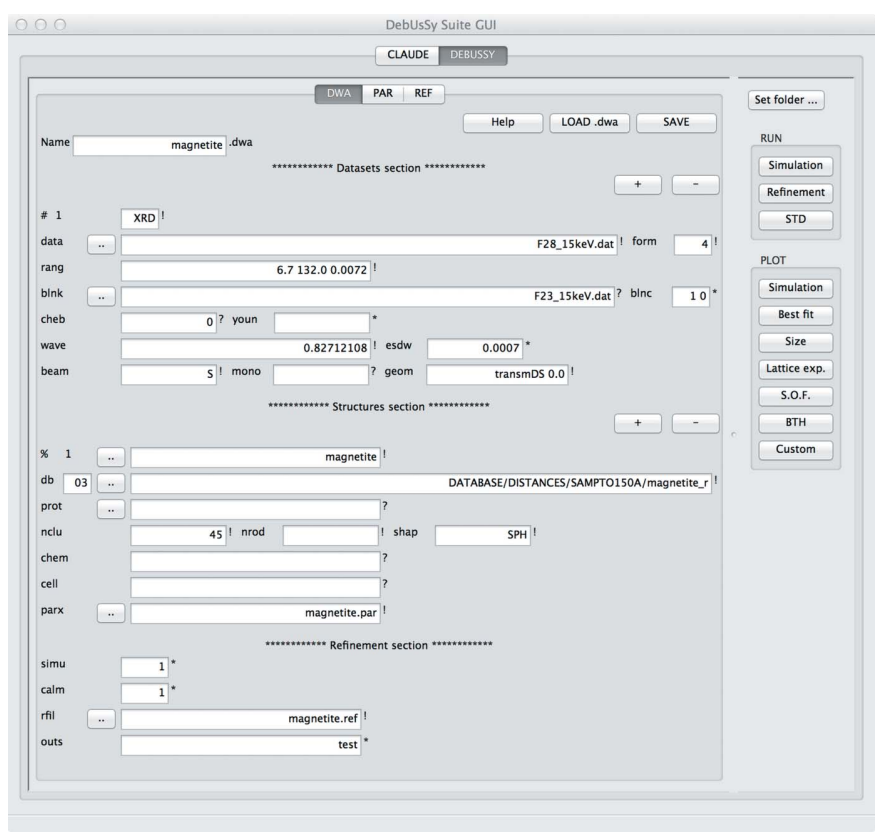

Figure 5

The DEBUSSY_GUI window with the editor of the main input file (.dwa) for data sets, structural models and refinement strategy selection.

user, instead of being hidden in the traditional menu-like style, with the aim of providing easier and therefore faster usage. As an example, in Fig. 4 the POPUlation DATABase tab is shown with the corresponding input parameters for the database generation.

The DEBUSSY_GUI is organized in a similar way, that is the GUI window contains three tabs to edit from scratch or load any of the main DEBUSSY input files (.dwa, .par and

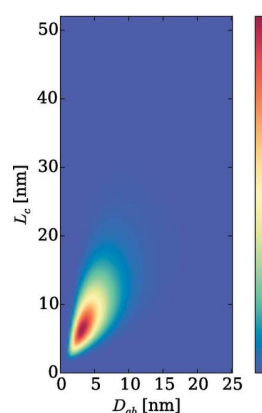

(a)

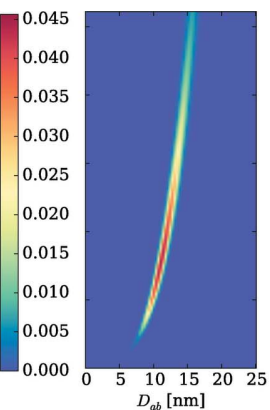

(b)

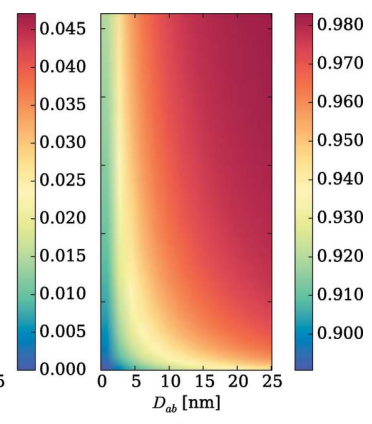

(c)
Figure 6

Two-dimensional maps of $(a),(b)$ the bivariate mass-weighted lognormal size distribution of nanocrystal population in two apatite samples of different sizes and $(c)$ the size-dependent site occupancy factor of the $\mathrm{Ca}$ atom in the same sample shown in (b) (Delgado-López et al., 2014).

.ref) and a vertical side panel collecting the RUN and PLOT buttons. Fig. 5 shows the DEBUSSY 2.0 DWA tab of the $D E B U S S Y \_G U I$ window. For a $D E B U S S Y$ run in simulation mode, filling the DWA and PAR tabs is mandatory, while for an optimization run also the REF tab must be filled with the refinement strategy parameters. DEBUSSY_GUI is used in a very similar manner to CLAUDE_GUI: once the necessary input parameters have been filled in and saved, clicking on any RUN-type button will launch the DEBUSSY program in the selected mode (simulation, refinement or standard error calculation). The PLOT-type buttons are instead used to generate automatic plots of some of the DEBUSSY output files (Fig. 6).

The DEBUSSY input files, options and execution procedure are described in detail in the manual, which is enclosed in the $D E B U S S Y$ package and accessible through the GUI window. The GUI code is written in Python 2.7.x and wxPython 2.8 using the classical class style of the Python language. The plotting functions use the standard Python plotting library Matplotlib (Hunter, 2007). DEBUSSY currently runs on Unixlike platforms (Linux and MacOSX included) and is available at http://debussy.sourceforge.net/debussy/debussy.html along with tutorial files.

\section{Acknowledgements}

Partial funding from Fondazione CARIPLO, projects 20092446 and 2011-0289, is acknowledged. FB acknowledges the University of Insubria for a Junior Fellowship Grant 2013.

\section{References}

Bertolotti, F., Maspero, A., Cervellino, A., Guagliardi, A. \& Masciocchi, N. (2014). Cryst. Growth Des. 14, 2913-2922.

Cernuto, G., Galli, S., Trudu, F., Colonna, G. M., Masciocchi, N., Cervellino, A. \& Guagliardi, A. (2011). Angew. Chem. Int. Ed. 50, 10828-10833.

Cernuto, G., Masciocchi, N., Cervellino, A., Colonna, G. M. \& Guagliardi, A. (2011). J. Am. Chem. Soc. 133, 3114-3119.

Cervellino, A., Frison, R., Cernuto, G., Guagliardi, A. \& Masciocchi, N. (2014). J. Appl. Cryst. 47, 1755-1761.

Cervellino, A., Giannini, C. \& Guagliardi, A. (2003). J. Appl. Cryst. 36, 1148-1158. 
Cervellino, A., Giannini, C. \& Guagliardi, A. (2006). J. Comput. Chem. 27, 995-1008.

Cervellino, A., Giannini, C. \& Guagliardi, A. (2010). J. Appl. Cryst. 43, 1543-1547.

Cervellino, A., Giannini, C., Guagliardi, A. \& Zanchet, D. (2004). Eur. Phys. J. B, 41, 485-493.

Cervellino, A. \& Guagliardi, A. (2010). Diffraction at the NanoscaleNanocrystals, Defective and Amorphous Materials, edited by A. Guagliardi \& N. Masciocchi. Varese: Insubria University Press.

Cervellino, A., Maspero, A., Masciocchi, N. \& Guagliardi, A. (2012). Cryst. Growth Des. 12, 3631-3637.

Cheary, R. W. \& Coelho, A. (1992). J. Appl. Cryst. 25, 109-121.

Debye, P. (1915). Ann. Phys. 351, 809-823.

Delgado-López, J. M., Frison, R., Cervellino, A., Gómez-Morales, J., Guagliardi, A. \& Masciocchi, N. (2014). Adv. Funct. Mater. 24, 1090-1099.

Egami, T. \& Billinge, S. J. L. (2003). Underneath the Bragg Peaks, Structural Analysis of Complex Materials. Oxford, New York: Pergamon, Elsevier.
Frison, R., Cernuto, G., Cervellino, A., Zaharko, O., Colonna, G. M., Guagliardi, A. \& Masciocchi, N. (2013). Chem. Mater. 25, 48204827.

Grover, R. F. \& McKenzie, D. R. (2001). Acta Cryst. A57, 739740.

Guagliardi, A., Cedola, A., Giannini, C., Ladisa, M., Cervellino, A., Sorrentino, A., Lagomarsino, S., Cancedda, R. \& Mastrogiacomo, M. (2010). Biomaterials, 31, 8289-8298.

Hall, B. D. \& Monot, R. (1991). Comput. Phys. 5, 414-417.

Hunter, J. D. (2007). Comput. Sci. Eng. 9, 90-95.

Metropolis, N., Rosenbluth, A. W., Rosenbluth, M. N., Teller, A. H. \& Teller, E. (1953). J. Chem. Phys. 21, 1087.

Navaza, J. (2002). Acta Cryst. A58, 568-573.

Nelder, J. A. \& Mead, R. (1965). Comput. J. 7, 308-313.

Rietveld, H. M. (1969). J. Appl. Cryst. 2, 65-71.

Thomas, N. W. (2010). Acta Cryst. A66, 64-77.

Welberry, T. R. (2010). Diffuse X-Ray Scattering and Models of Disorder. International Union of Crystallography Monographs on Crystallography. Oxford University Press. 